\title{
Arthroscopic Meniscal Transplantation Technique
}

\section{Cruz-Lopez Francisco, Olivos-Meza Anell, Llano Rodríguez Luis Tomas and Ibarra Clemente*}

National Institute of Rehabilitation, Mexico

\begin{abstract}
The meniscus provide several important mechanical functions in the knee joint. Following menisectomy the tibiofemoral contact area decreases while the contact forces increase. Meniscal allograft transplantation (MAT) offers the potential to restore partial load-bearing, decrease symptoms, and provide chondroprotective effect. Ideal patient for MAT should have normal alignment, stable knee, abscence of cartilage damage and has less than 50 years-old. Several open and arthroscopic MAT techniques have been described. The succesful of MAT is to use a minimal invasive technique without sacrificing the precise anatomic reconstruction of the original meniscofemoaral and meniscotibial relationships. Anatatomical position, appropriate sizing of the graft, and fixation method are crucial key points to have good results. In general there are three fixation methods: soft tissue fixation, suture fixation through transoseous tunnels, and bone plugs press-fit fixation. However, inferior biomechanical and contact pressure have been reported with soft tissue and transosseous tunnels compared with the bone block technique.
\end{abstract}

Keywords: Surgical techniques; Sports medicine; Surgery; Meniscal transplantation

\section{Introduction}

Meniscus play an important role in load transmission, stability, lubrication and nutrition of the articular cartilage. Lateral meniscus has a greater role in stress protection and abscense of this structure leads to higher incidence of osteoarthrosis while medial meniscus is more important in joint stability [1]. Meniscal pathology remains a common entity in the knee. In complex and chronic injuries, meniscal preservation is not possible. Following menisectomy, tibiofemoral contact area decreases by approximately $50 \%$, while contact forces increase 2 -fold to 3 -fold and in consequence osteoarthric changes in the kne are well documented $[2,4]$. Allograft transplantation technique offers the potential to restore function, provides chondroprotective effects, and decrease symptoms in appropriate patients after total menisectomy [2]. Ideal patient to meniscal transplantation is young (less than fifty years), stable knee, normally aligned and localized pain in the affected compartment [4-6]. Contraindications include inflammatory arthritis, septic arthritis, immunodeficiency, obesity, systemic metabolic diseases, skeletal immadurity, advanced osteoarthritis (grade III or IV), and flattening of the femoral condyle. There are relative contraindications to meniscal transplantation as ligamentous instability, malalignment, and cartilage degeneration. Prophylactic meniscus transplantation is not recommended in asymptomatic patients who do not have articular cartilage damage.

Altough clinical results are promising, the use of meniscal allograft transplantation should be considered a salvage operation in young patients. Numerous open and arthroscopic techniques have been described; however the most importants issues in meniscal transplantation are anatatomical position and appropriate sizing of the graft. One controversial and crucial aspect is the fixation of the meniscal horns of the graft to make sure they can withstand loading. Bone anchoring of the meniscal horns provides greater biomechanical properties and better stability than fixation to soft tissues [7].

The goal of this study is to demonstrate the feasibility of arthroscopic meniscal transplantation using press-fit bone fixation and graft placement using tibial tunnels.

\section{Technical note}

This meniscal allograft technique is used in a research protocol at the National Institute of Rehabilitation in Mexico city. Patients were recluted in the Orthopedic Sports Medicine Service in a period of seven years. Inclusion criteria were: age (18-50 years old), history of total meniscectomy, post-meniscectomy syndrome, focalized chondral lesions (Outerbridge III, IV) that can be treated by ACI or Microfracture Techniques simultaneously knee stability and well alignement.

The study was approved by the local ethics committee of the hospital, and all procedures were performed by the same orthopedic surgeon trained in arthroscopic knee surgery with more than 10 years of experience.

\section{Materials and Methods}

\section{Surgical techniques}

The preparation of the host meniscus remanent is crucial; is important not to remove completely the meniscal rim in order to provide an excelent bed for allograft fixation. The patient is placed supine, involved leg is positioned with a well-padded leg holder. The contralateral leg is also well-padded to prevent tension on the femoral nerve and fixed in abduction position.

Before preparation, meniscus allograft is immersed in sterile saline solution with Gentamicin antibiotic $(160 \mathrm{mg})$ during 30 minutes. Soft tissue capsular remnants and excess tissue around the anterior and posterior horns are excised. The bone bridge is sized and prepared according to the tibial slot or 2 bone blocks for medial or lateral meniscus, respectivelly. A mattress 2-0 Orthocord suture is placed under the bonny component of the anterior and posterior horn in order to settle the bone bridge or bone blocks into the tibial surface. All-inside technique is used for posterior horn; inside-out vertical suturing is used to hold in place the meniscal body, while the anterior horn is fixed with an outside-in technique. Routine diagnostic arthroscopy is performed through standard arthroscopic portals.

*Corresponding author: Ibarra Clemente, Calzada Mexico Xochimilco 289 Arenal de Guadalupe, Mexico city. ZC 14389, Tel: 05259991000, Ext. 19607; Fax: 59-99-10-00; E-mail: cibarra.corresponding@hotmail.com

Received October 09, 2015; Accepted December 07, 2015; Published December 11,2015

Citation: Francisco CL, Anell OM, Tomas LRL, Clemente I (2015) Arthroscopic Meniscal Transplantation Technique. J Sports Med Doping Stud 6: 171. doi:10.4172/2161-0673.1000171

Copyright: (c) 2015 Francisco CL et al. This is an open-access article distributed under the terms of the Creative Commons Attribution License, which permits unrestricted use, distribution, and reproduction in any medium, provided the original author and source are credited. 


\section{Allograft preparation: lateral meniscus}

Before preparation, meniscus allograft is immersed in sterile saline solution with Gentamicin antibiotic (160mg) during 30 minutes. Soft tissue capsular remnants and excess tissue around the anterior and posterior horns is excised. Then, allograft and bone bridge are sized according to preoperative plain film radiographs and operative arthroscopic meassurements.

With a thin and small oscilating saw the bone bridge is sized to a width of 6 or $7 \mathrm{~mm}$ and height $1 \mathrm{~mm}$ less than the tibial slot height (Figure 1A and 1B). Care must be taken to avoid damage to the meniscus and its insertions. With a Kirschner nail (0.045 diameter) two vertical holes were perforated in the anterior and posterior third of the bone bridge in order to pass a mattress orthocord suture (Figure 1C). Then a third longitudinal suture is passed over the bone bridge through one anterior and one posterior holes, this is very useful to seat and fix the anterior and posterior borders of the bone plug under the tibial plateau bridge.

A further vertical mattress suture is placed through the junction of the posterior and midle third of the meniscus. Also a couple of vertical perforations throug meniscus tissue are done every $5 \mathrm{~mm}$ from the posterior horn to anterior horn, those are reference marks to facilitate arthroscopic meniscal fixation.

\section{Lateral meniscus transplantation}

After diagnostic arthroscopy, the remnant meniscus is resected leaving 1-2 $\mathrm{mm}$ of the meniscal capsular rim. Through the lateral portal, the lateral tibial spine is flatened with a burr (Figure 2A). A superficial reference slot is made on the tibial plateau in the anteroposterior direction (Figure 2B). A small portion of the medial border of the lateral femoral condyle is resected to improve posterior visualization and facilitate the graft passage.

The lateral portal is extended and become the lateral parapatellar mini-arthrotomy. An alignment rod is positioned over the superficial mark reference, taking care that it lies directly over the insertion of anterior and posterior horns. A 5 millimeters bone spurr is then used to deep and smooth the slot to facilitate the allograft bone bridge insertion. A curet is used to clear any remaining bone in the walls of the slot and flattening the floor. The lenght, depth, and width are measured to ensure the proper bone block size. An ACL guide is used to place through the center of insertion sites a $2.4 \mathrm{~mm}$ guide pin (Figure $2 \mathrm{C}$ and $2 \mathrm{D}$ ). Once the guide pin is well placed, the posterior tibial tunnel is drilled using a 7-8 $\mathrm{mm}$ reamer. Same step is repeated to create the anterior horn tunnel as perpendicular as possible to the tibial plateau. The allograft and bone block are inserted into the lateral comparment through mini-arthrotomy with the bone block seated into into the tibial canal while varus force is applied to open the joint space. The passing vertical sutures through the bone plug should position the graft properly while the third horizontal help to bring down into the tibial slot the meniscus bone graft (Figure 3A).

All-inside (Fast-Fix Suture System, Smith \& Nephew) technique is used for posterior horn fixation (Figure 3B); inside-out vertical suturing with PDS is used to hold in place the body of the meniscus allograft (Figure 3C and 3D) while the anterior horn is fixed with an outside-in technique using a couple of 18 gauge spinal needles and PDS sutures.

\section{Allograft preparation: medial meniscus}

The bone block is cut to a depth of $10 \mathrm{~mm}$. An $8 \mathrm{~mm}$ coring reamer is used to cut the bone plug out of the bone block for anterior and

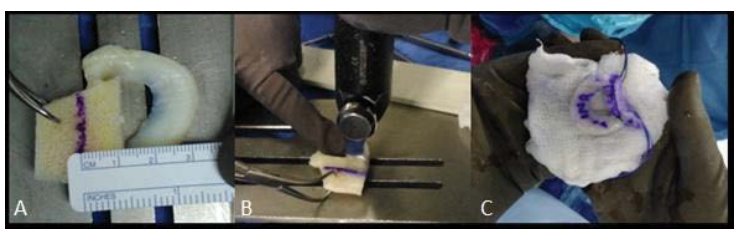

Figure 1: Preoperative photograph demostrating a meniscus allograft preparation; (A, B): Sizing and cutting of the bone bridge. (C) Allograft prepared with mattress orthocord suture.

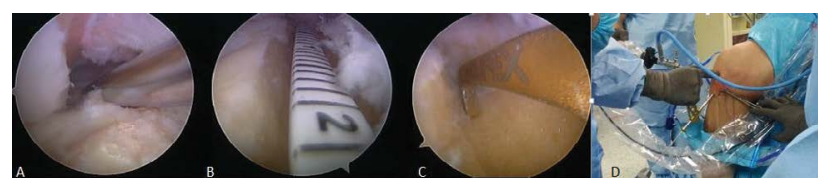

Figure 2: Arthroscopic images of a lateral meniscus transplantation. (A) Lateral tibial spine flattened with a burr. (B) Measure of the tibial slot. (C) Localization for posterior tibial tunnel drill. (D) Intraoperative photograph demostrating the use of the ACL guide.

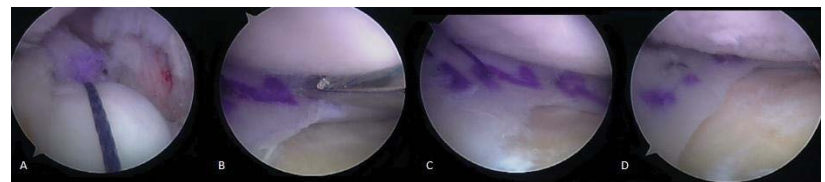

Figure 3: Arthroscopic images of lateral meniscus transplantation. (A) Insertion of the allograft and bone block into the tibial slot, showing the passing vertical sutures. (B) Fixation of the allograft to the meniscus remnant and capsule using an all inside technique. (C) Fixation of the allograft to the capsule using an inside-out technique. (D) Final view of a lateral meniscus allograft transplant.

posterior horns, finally the bone plug is fashioned with a ronguer. Highstrength orthocord suture is passed through the bottom of anterior and posterior plugs. Those sutures will later be used to secure the bone plugs through the tibial tunnels.

\section{Medial meniscus transplantation}

Patient positioning, preparation, and diagnostic arthroscopy for the medial meniscus are the same as for the lateral meniscus. The medial meniscus transplant we use is the double bone plug procedure. The medial portal is our working portal and is very important to line it up with the anterior and posterior horn insertion sites. The native tissue is cutting and a 1 to $2 \mathrm{~mm}$ remnant is left (Figure $4 \mathrm{~A}$ ). Insertion sites of anterior an posterior horns are identified. An ACL guide is used to place through the center of inertion sites a $2.4 \mathrm{~mm}$ guide pin. Once the guide pin is well placed, the posterior tibial tunnel is drilled using a 7-8 $\mathrm{mm}$ reamer. Same step is repeated to create the anterior horn tunnel as perpendicular as possible to the tibial plateau. Working portal extended and became a mini-arthrotomy; meniscus allograft is placed in the medial compartment through this arthrotomy. The posterior bone plug is positioned into the posterior tibial tunnel by suture traction while a blunt instrument is used to set the allograft in place (Figure 4B). The anterior bone plug is reduced into the tunnel with a press fit technique and with suture traction (Figure 4C). The knee is moved from flexion to extention to confirm the position of the allograft.

All-inside (Fast-Fix Suture System, Smith \& Nephew) technique 
is used for posterior horn fixation (Figure 5A); inside-out vertical suturing with PDS is used to hold in place the body of the meniscus allograft (Figure 5B \& 5C) while the anterior horn is fixed with an outside-in technique using a couple of 18 gauge spinal needles and PDS sutures (Figure 6).

\section{Rehabilitation}

Rehabilitation programs after meniscal transplantation is similar to those for meniscal repair. The patient is kept non-weight-bearing for the first 4 weeks after surgery; partial weight bearing in extension is initiated only at 5 weeks, and full weight bearing at 7 weeks. Range of motion begins the day after surgery but is limited to $0^{\circ}$ to $90^{\circ}$ during 4 weeks. The recommendation to the patient is not intended to return to intense sports or high impact activities that involve jogging, running, or jumping.

\section{Results}

Our workgroup has performed thirty-five arthroscopic meniscal allograft transplantation with bone plug press-fit fixation combined with soft tissue suture fixation. Sixteen patients were women (46\%) while nineteen were men (54\%). Patients mean age was 32.4 years-old (19 to 50). All surgical procedures have been performed in a period of 7 years by the same surgeon (2008 to 2015). Fiffteen MAT were medial $(80 \%)$ and twenty $(57 \%)$ were lateral. Both, fresh frozen $(57 \%)$ and gamma irradiated (43\%) grafts were transplanted. All operated patients received Magnetic Resonance Imaging (MRI) after surgery (every twelve-months of follow-up). Studies showed capsular graft integration and any graft extrussion.

Clinically, we observed significant improvements in pain and function between scores applied before and after MAT in both groups.

\section{Discussion}

Meniscal allograft transplantation is a well-established procedure that nowadays is performed all over the world. Meniscal Allograft Transplantation (MAT) is a viable option in patients with symptoms after subtotal or total meniscectomy. Multiple studies have shown improvement in quality of life, and activities of daily living. MAT can be performed with either an open or arthroscopic approach, but the

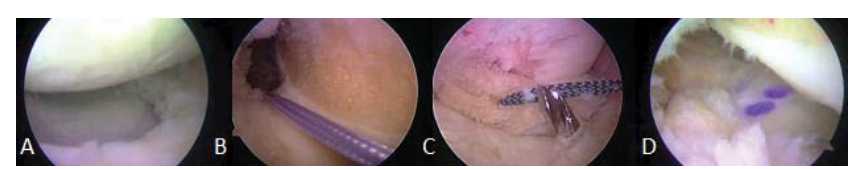

Figure 4: Arthroscopic images of medial meniscus transplantation. (A) Preparation of the native tissue leaving a 1 to $2 \mathrm{~mm}$ of meniscus remnant. (B) Position of the posterior bone plug in the posterior horn tunnel by suture traction. (C) Drilling of the anterior horn tunnel. (D) Passing of the anterior bone plug through the anterior horn tunnel by suture traction.

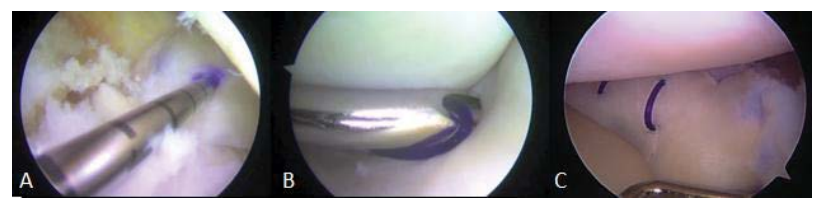

Figure 5: Arthroscopic images of medial meniscus transplantation. (A) posterior horn fixation using an all inside technique with a Fast-Fix suture system. $(B, C)$ Meniscal body fixation using an inside-out, and an outside-in technique.

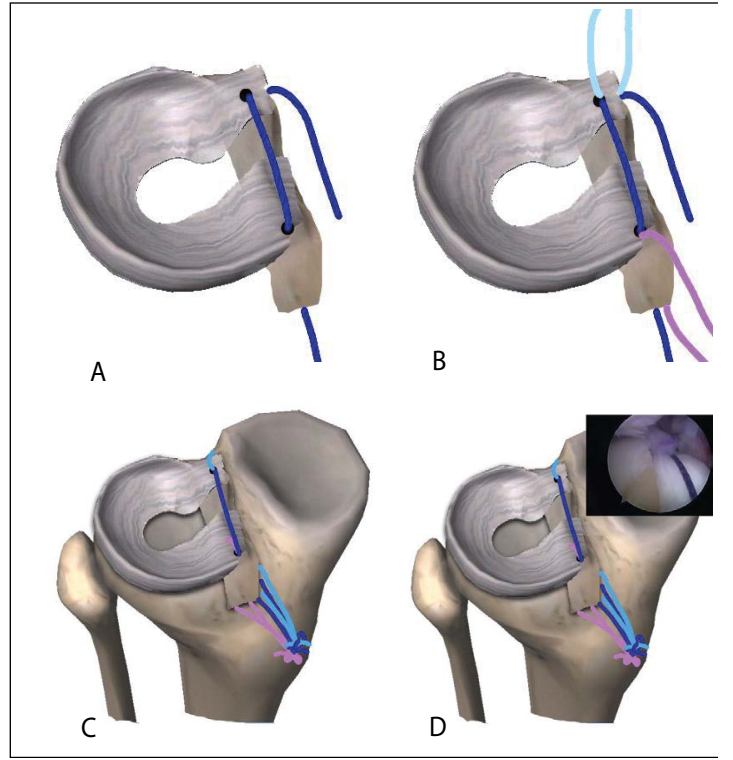

Figure 6: Lateral meniscal transplant fixation. (A) Longitudinal suture over the slot to seat the bone graft in the ridge. (B) Anterior and posterior sutures to hold meniscal horns. (C) Three sutures are passed through bone tunnels and fixed with knots over the anterior cortical of the tibia. (D) Arthroscopic view of the bone slot perfectly seated into the ridge.

latter is more widely used recently.

Arthroscopic meniscal transplantation reduces surgical morbidity being a safety procedure; although technically challenging warrants a reproducible technique that permits reliable anatomic placement of either medial or lateral meniscal allograft. Similar results have been reported in the post-operative period in patients undergoing open and arthroscopic meniscal transplantation; cosmetic issue is the only difference between techniques [8,9]. However, arthroscopic based procedures avoided collateral ligament disruption, reduce surgical morbility, and promotes earlier rehabilitation [10-13]. This technique also facilitate the treatment of concomitant injuries as chondral lesions and ligament instability of the knee. Chondral injuries are commonly encountered in post-menisectomized patients and its treatment is very important.

Although the succes of the procedure depends on multiple patient related and surgical factors. Wheter an arthroscopic or open technique is selected for meniscal transplantation, the correct anatomic placement is the important surgical issue. The transplantation of a meniscus as a free graft was developed in Germany in the mid-1980s and it became popular as a simple method of meniscus transplant without any bone block; this was a way to simplyfy the fixing technique, by securing the graft with sutures alone to the soft tissue. However, current biomechanical data have provided controversial information relative to the superiority of soft tissue technique versus bone fixation, last one offers to restore normal tibial forces and distribute the loading to protect articular cartilage [14-16]. McDermott et al. Showed that lateral meniscal allografts that were only fixed with sutures made higher articular cartilage pressure that when they had bone fixation [17] Hunt et al. Tested the necessity to fix the posterior horn of the medial transplant fixation in regard to using sutures alone versus bone plug plus sutures. Other experimental studies have shown good healing of the transplanted allograft when both horns are sutured without bone plug fixation. As a conclusion, no strong evidence currently exist to 
show the biomechanical advantage of either of the two twchniques.

Open meniscus allograft transplantation has the ability to preinsert all peripheral sutures apparently in a more accurate and less traumatic manner. However, with the new arthroscopic implants for meniscal suture have minimal possibility to damage articular cartilage. Arthroscopic MAT not only provides smallest incisions but also remains medial collateral ligament intact in the case of medial meniscal transplant [18].

Time procedure spent with arthroscopic technique was between 1.5 to 2 hours, a reasonable time to reduce surgical complications [12]. In a magnetic resonance imagine study comparing open versus arthroscopic meniscus transplant, De Coninck et al. Reported that radial displacement of MAT arthroscopically inserted with bone tunnel fixation was significantly less than the radial displacement of MAT onserted with open soft-tissue fixation [19].

\section{Conclusion}

This is an arthroscopic technique that permits either medial and lateral meniscal allograft transplantation with combined fixation (bone and soft tissue fixation), having the benefits of leave anterior and posterior horns anatomically placed with the benefits of being a less invasive surgical procedure.

\section{Acknowledgement}

The authors wish specialty to thank to Biograft of México Musculoeskeletal Tissue and Skin Bank for the donation of meniscal allografts.

\section{Conflict of Interest}

All authors have equal contribution to the manuscript.

\section{References}

1. Van Arkel ER, de Boer HH (1995) Human meniscal transplantation. Preliminary results at 2 to 5-year follow-up. J Bone Joint Surg Br 77: 589-595.

2. Noyes FR, Heckmann TP, Barber-Westin SD (2012) Meniscus repair and transplantation: a comprehensive update. J Orthop Sports Phys Ther 42: 274 290.

3. Elliot D, Jones R, Setton L, Scully S, Vail P, et al. (2001) Joint Degeneration following meniscal allograft Transplantation in Canine Model: Mechanical Properties and Semiquantitative Histology of Articular Cartilage. Knee Surg Sports Traumatol Arthrosc 10: 109-118.
4. Shybut T, Strauss EJ (2011) Surgical management of meniscal tears. Bull NYU Hosp Jt Dis 69: 56-62.

5. Kelly BT, Brophy RH, Rodeo SA (2004) Meniscal allograft transplantation: surgical technique. Tech Knee Surg 3: 1-11.

6. Verdonk R, Almqvist F (2002) Meniscal transplantation. Techinques in Knee Surgery 1: 23-35

7. Alvarez P, Cusco X (2010) Meniscal transplant technique without bone blocks Techinques in Knee Surgery 9:165-169

8. Lee AS, Kang RW, Kroin E, Verma NN, Cole BJ (2012) Allograft meniscus transplantation. Sports Med Arthrosc 20: 106-114.

9. Khetia EA, McKeon BP (2007) Meniscal allografts: biomechanics and techniques. Sports Med Arthrosc 15: 114-120.

10. Verdonk PC, Demurie A, Almqvist KF, Veys EM, Verbruggen G, et al. (2005) Transplantation of viable meniscal allograft. Survivorship analysis and clinical outcome of one hundred cases. J Bone Joint Surg Am 87: 715-724.

11. Noyes FR, Barber SD, Marc Rankin (2005) Meniscal transplantation in symptomatic paients less than fifty years old. J Bone Joint Surg Am 87:146165.

12. Rodeo SA1 (2001) Meniscal allografts--where do we stand? Am J Sports Med 29: 246-261.

13. Goble EM (2004) Arthroscopic meniscus transplantation: plug and slot technique. In: Miller M, Cole BJ (edn) Text-book of arthroscopy. WB Saunders Philadelphia, USA.

14. Cole BJ, Carter TR, Rodeo SA (2003) Allograft meniscal transplantation: background, techniques, and results. Instr Course Lect 52: 383-396.

15. Rijk PC1 (2004) Meniscal allograft transplantation--part I: background, results graft selection and preservation, and surgical considerations. Arthroscopy 20 728-743.

16. Dave Lee YH, Caborn D (2012) A New Technique for Arthroscopic Meniscus Transplantation Using Soft Tissue Fixation and Anatomical Meniscal Roor Reinsertion. Knee Surg Sports Traumatol Arthrosc 20: 904-908.

17. Jang SH, Kim JG, Shim JC (2011) Reducing the size of the meniscal allograft decrease the percentage of extrusion after meniscal allograft transplantation. Arthroscopy 27: 914-922.

18. Goble EM, Verdonk R, Kohn D (1999) Arthroscopic ans Open Surgical Techniques for Meniscus Replacement-Meniscal Allograft Transplantation and Tendon autograft Transplantation. Scand J Med Sci Sports 9: 168-176.

19. De Coninck T, Huysse W, Verdonk R, Verstraete K, Verdonk P (2013) Open versus arthroscopic meniscus allograft transplantation: magnetic resonance imaging studi of meniscal radial displacement. Arthroscopy 29: 514-21. 